\title{
Effects of chlorhexidine, essential oils and herbal medicines (Salvia, Chamomile, Calendula) on human fibroblast in vitro
}

\author{
MARZENA WYGANOWSKA-SWIATKOWSKA ${ }^{1}$, PAULINA URBANIAK ${ }^{2}$, ANNA SZKARADKIEWICZ ${ }^{1}$, \\ JERZY JANKUN ${ }^{3}$, MALGORZATA KOTWICKA ${ }^{2}$
}

${ }^{1}$ Department of Conservative Dentistry and Periodontology, Poznan University of Medical Sciences, Poznan, Poland

${ }^{2}$ Department of Cell Biology; Poznan University of Medical Sciences, Poland

${ }^{3}$ Urology Research Center, Department of Urology, College of Medicine, University of Toledo, Toledo, USA

\begin{abstract}
Antiseptic rinses have been successfully used in inflammatory states of the gums and oral cavity mucosa. Antibacterial effects of chlorhexidine, essential oils and some herbs are well documented. Reaction of host tissue to these substances has much poorer documentation. The aim of the study was to analyse the influence of chlorhexidine (CHX), essential oil (EO: thymol, 0.064\%; eucalyptol, 0.092\%; methyl salicylate, $0.060 \%$; menthol, $0.042 \%$ ) mouth rinses and salvia, chamomile and calendula brews on fibroblast biology in vitro. The human fibroblast CCD16 line cells were cultured in incubation media which contained the examined substances. After 24 and 48 hours, the cell morphology, relative growth and apoptosis were evaluated. Exposure of fibroblasts to $C H X, E O$ or salvia caused various changes in cell morphology. Cells cultured for 48 hours with CHX revealed a noticeably elongated shape of while cells cultured in high EO concentration or with salvia were considerably smaller and contracted with fewer projections. Chlorhexidine, EO and salvia reduced the fibroblast proliferation rate and stimulated cell death. Both reactions to EO were dose dependent. Cells exposure to chamomile or calendula brews did not change morphology or proliferation of fibroblasts. The results of this in vitro study showed that in contrast to chamomile and calendula, the brews of EO, CHX or salvia had a negative influence on fibroblast biology.
\end{abstract}

Key words: mouth rinses, chlorhexidine, essential oils, herbs, fibroblast, morphology, proliferation.

(Cent Eur J Immunol 2016; 41 (2): 1-7)

\section{Introduction}

Inflammation and destruction of periodontal tissues are considered to result from response of susceptible host tissue to the microbial biofilm containing gram-negative bacteria pathogens present in the oral cavity [1]. The subgingival environment is one in which those pathogens flourish. Changes in tissue of the periodontal pocket or in supragingival plaque lead to changes in the composition of the subgingival plaque. Because the supragingival plaque removal can decrease periodontal pathogens, mechanical plaque removal is the basis of most periodontal treatment regimens by professionals and at home. The optimal result was observed in non-surgical elimination of plaque from periodontal pockets in combination with chemical prophylaxis $[2,3]$. The antibacterial effect of chlorhexidine, triclosan, cetylpyridinium chloride, iodine, essential oils and natural antiseptics is well documented $[4,5]$. Antiseptics are also commonly used in anti-malodour efficacy, sub- gingival irrigation and in the early postoperative phase. However, the reaction of host tissues to their influence has been poorly documented [6].

In the group of antiseptics the essential oils and herbs are believed to have the lowest tissue toxicity, especially at the healing stage $[7,8]$. Wound healing is a dynamic process involving the coordinated action of both resident and migratory cell populations within the extracellular matrix (ECM) and cytokines. The biologic control of ECM synthesis by fibroblasts at the wound site is a complex process depending upon the matrix constituents within the wound (collagen types I, III, V, fibronectin and glycosaminoglycans) and cytokines/growth factors produced by inflammatory cells, keratinocytes and fibroblasts themselves. Therefore, fibroblasts play a pivotal role in tissue repair as, by their proliferation and ECM synthesis, they control collagen deposition at the wound site. They play a vital role in the maintenance of healthy periodontium $[1,9,10]$.

Correspondence: Marzena Wyganowska-Swiatkowska PhD, DDS, Department of Conservative Dentistry and Periodontology,

Collegium Stomatologicum, 70 Bukowska str. 60-812 Poznan, mobile: +48 601883 198, e-mail: marzena.wyganowska@periona.pl 
The aim of the study was to analyse the influence of chlorhexidine (CHX), essential oil (EO) mouth rinses and salvia, chamomile and calendula brews on human fibroblast biology in vitro to identify the less cytotoxic agents.

\section{Materials and methods}

\section{Mouthwashes}

In each experiment, commercially available mouthwashes containing $0.1 \% \mathrm{CHX}$ or EO containing a fixed combination of 4 essential oils (thymol, $0.064 \%$; eucalyptol, $0.092 \%$; methyl salicylate, $0.060 \%$; menthol, $0.042 \%$ ) were used. Herbal brews of salvia, chamomile and calendula were made in a traditional way: 2 grams of each herb was brewed in distilled water at the temperature of $90^{\circ} \mathrm{C}$ and then boiled under cover for 10 minutes. The solutions were left in sterile, tightly closed containers until they cooled down.

\section{Cell culture}

All experiments were conducted on non-malignant human fibroblasts cell line CCD16 (American Type Culture Collection; USA). CCD16 cell line was transferred in aseptic conditions from freezing medium RPMI 1640 (Gibco; USA), 30\% Fetal Bovine Serum (FBS; Gibco), $10 \%$ DMSO (Gibco) to $90 \mathrm{~mm}$ sterile petri dish (Sarstedt, Germany) containing $10 \mathrm{ml}$ of growth medium with the following composition: RPMI-1640 medium, 10\% FBS, antibiotics: penicillin $100 \mathrm{mg} / \mathrm{ml}$ and streptomycin 100 $\mathrm{mg} / \mathrm{ml}$ (Gibco) and $2 \mathrm{mmol} / \mathrm{l} \mathrm{L-glutamine} \mathrm{(Gibco).} \mathrm{Cells}$ were grown in aseptic conditions, in incubator with controlled temperature of $37^{\circ} \mathrm{C}, 5 \% \mathrm{CO}_{2}$ and $100 \%$ humidity conditions.

Cells were cultured until they formed a monolayer. After having received $90 \%$ confluence the cells were detached with trypsin-EDTA $(0.25 \%$ trypsin containing $0.01 \%$ EDTA) solution. After 5 minutes of incubation, complete growth medium was added to inhibit the reaction. Cell suspension was transferred to petri dishes and the culture medium was added at the volume ratio of 1/10. Cells prepared in this procedure were used for further investigations.

\section{Effects of rinses on fibroblast proliferation}

On a day prior to rinses stimulation cells were passaged to $35 \mathrm{~mm}$ petri dishes, with the cell number of $4 \times$ $10^{5}$ per plate. $1.25 \mathrm{ml}$ of cell suspension in the culture medium was supplemented with $250 \mu$ of each rinse. In each experiment CHX $0.1 \%$, essential oils in concentration of $10 \%$ (EO 10), $50 \%$ (EO 50) or $100 \%$ (EO 100) of the commercially available concentration, salvia, chamomile and calendula brews were used. $10 \%$ of ethyl alcohol solution was used as a control for $\mathrm{CHX}$ and EO (both CHX and EO are alcohol-based preparations; $0.1 \% \mathrm{CHX}$ contains $7.2 \%$ of the alcohol whereas EO contains $22 \%$ of ethyl alcohol).
The morphological observations were made under the Diaphot Eclipse TC (Nikon) microscope. On each plate cells were counted within three $2.54 \mathrm{~mm}^{2}$ areas, marked with an ink objective marker (Nikon). For morphological characterization of the dead cells, fluorescent dyes were used after 48 hours of cell incubation. Early signs of apoptosis in intact cells at the level of chromatin structure (chromatin condensational and fragmentation) were visualized with $0.1 \mu \mathrm{g} / \mathrm{ml}$ Hoechst 33342 dye (excitation at $365 \mathrm{~nm}$, emission above 510 $\mathrm{nm}$; Sigma, Germany). Permeabilization of the plasma membrane, occurring during necrosis or late phases of apoptosis, was found with propidium iodide stain (excitation at 530-560 $\mathrm{nm}$, emission above $580 \mathrm{~nm}$; Sigma, Germany).

The relative increase in the cell numbers $(\mathrm{Rn})$ after 24 and 48 hours of incubation was calculated according to the following formula: $\mathrm{Rn}=(\mathrm{Tn} / \mathrm{To}) \times 100 \%$; $\mathrm{Tn}-$ the number of cells after 24 or 48 hours; To - the number of cells at the beginning of the experiment.

\section{Statistical analysis}

For statistical analysis, GraphPad InStat software was used. U-Mann-Whitney and Kruskal-Wallis nonparametric test (with Dunn's multiple comparisons test) was used. $P<$ 0.05 was considered significant.

\section{Results}

\section{Fibroblast morphology}

The control cells were characterised by a high diversity of shapes. Usually there were elongated cells with several projections (Fig. 1A). The fibroblast nucleus was big, round or oval, with nuclear chromatin with granular structure with two nucleoli clearly seen (Fig. 1B). No change of cell morphology was noticed after 24 or 48 hours.

In comparison to the control group, the fibroblasts cultured for 24 hours in the incubation medium with the addition of alcohol contain a smaller number of projections and after 48 hours their morphology did not differ substantially from the control cells (images not shown).

The fibroblasts cultured with CHX for 48 hours were considerably elongated (Fig. C); they had few narrow projections. The strongly condensed granular nucleic chromatin structures were observed (Fig. 1D).

The morphology of cells cultured with EO 10 did not significantly change both after 24 and 48 hours. The cells cultured for 48 hours with EO 50 or EO 100 were considerably contracted with narrow projections (Fig. 1E); many dead cells were observed (Fig. 1F).

The addition of salvia to the medium significantly changed the cell morphology - a considerable part of the fibroblasts was contracted, they had few long and narrow projections (Fig. 1G). The cells presenting chromatin changes typical for apoptosis were observed (Fig. 1H). 

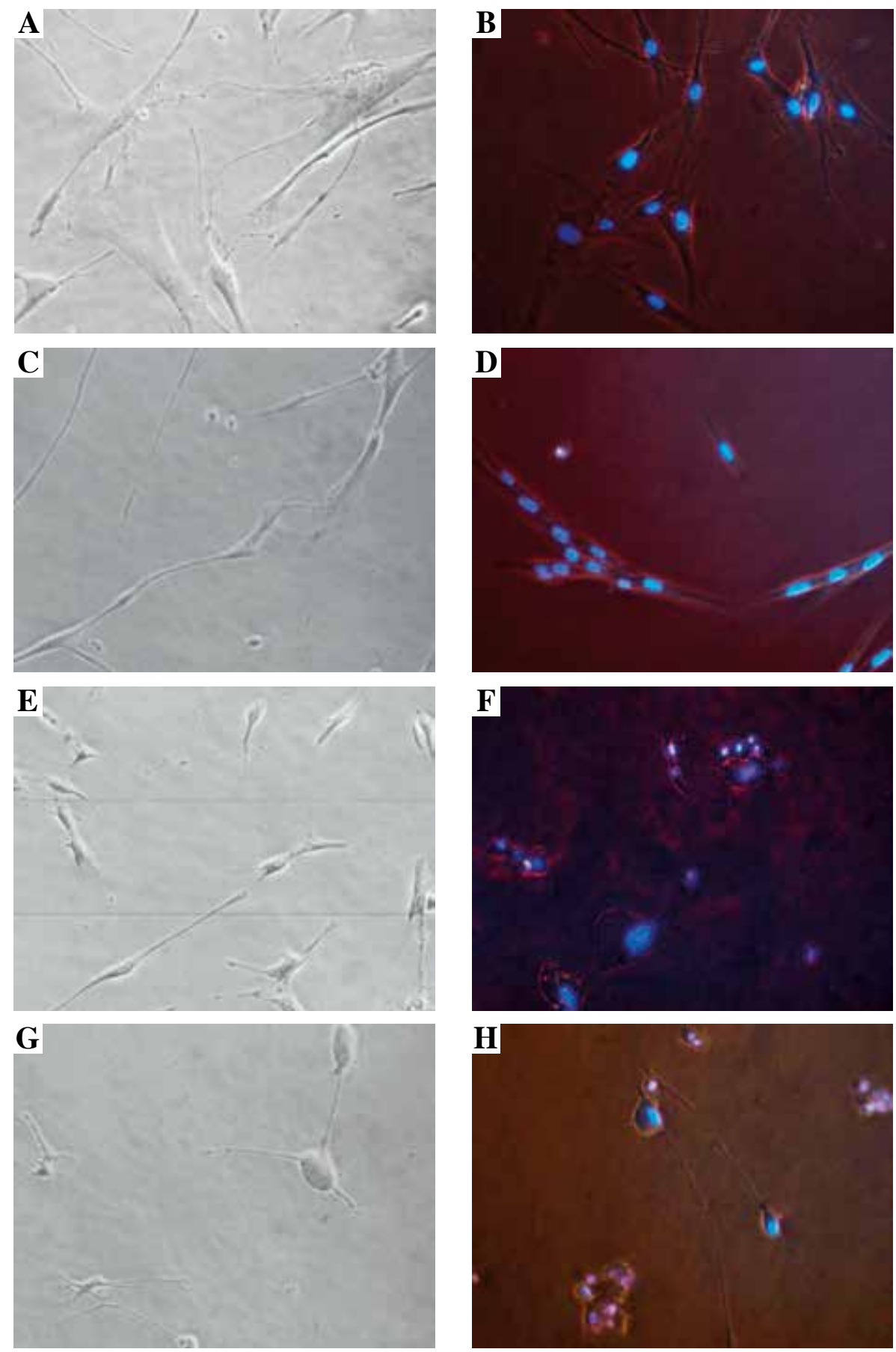

Fig. 1. The morphology of fibroblasts line CCD16. A and B: The fibroblasts in the control group were characterised by high diversification of shapes. The centrally located nucleus revealed the granular structure of nucleic chromatin with two nucleoli. C and D: The morphology of fibroblasts after 48-hour-culture in medium with CHO $0.1 \%$ : most cells were elongated, demonstrating isolated projections with strongly condensed granular nucleic chromatin structure. E and F: The morphology of fibroblasts after 48-hour-culture in medium with essential oils: cells were considerably contracted with narrow projections; many dead cells were observed (F). $\mathrm{G}$ and $\mathrm{H}$ : The morphology of fibroblasts after 48-hour-culture in medium with salvia brew: most cells were round-shaped and had isolated narrow projections; the increasing number of apoptotic and necrotic cells was observed $(\mathrm{H})$. A, C, E, G - no staining cells. B, D, F, H - cells stained with a mix of propidium iodine dye (dead cells - pink nucleus fluorescence) and Hoechst 33342 (living cells - blue nucleus fluorescence); microscope magnification $20 \times$ 
The morphology of chamomile stimulated cells did not significantly change. The cells incubated in the culture medium with a calendula solution were characterised by well-developed and numerous projections (images not shown).

\section{Fibroblast proliferation}

In the dishes with the control group cells, the initial number of cells was considered as $100 \%$. After $24-$ hour culture, the number of cells increased to 112.4 $\pm 1.3 \%$, whereas after 48 hours, it reached $137.4 \pm 5.8 \%$ $(p<0.001)$. Control cells exhibited normal staining with Hoechst 33342 dye. Isolated dead cells were observed.

In comparison with the control group, the 24-hour-culture of fibroblasts in the medium with alcohol did not significantly change the relative growth of cells in the dishes: $110.2 \pm 10.3 \%$ vs $112.4 \pm 1.3 \%, p>0.05$. After 48-hour-culture the increase in the number of cells was smaller than in the control group, but the difference was not statistically significant $(121.3 \pm 12.5 \%$ vs $137.4 \pm 5.8 \%$; $p=0.06)$. Isolated dead cells were observed.

\section{Effects of chlorhexidine on fibroblast proliferation}

Chlorhexidine caused a gradual decrease in the number of cells in the cell culture. In comparison with the control group, both after $24(85.1 \pm 10.3 \%$ vs $112.4 \pm 1.3 \%$, $p=0.0007)$ and 48 hours $(75.9 \pm 12.9 \%$ vs $137 \pm 5.8 \%$, $p=0.002$ ) of culture the difference was significant. After 48 hours of incubation, $8.9 \pm 1.9 \%$ of cells demonstrated apoptotic changes (chromatin condensation and fragmentation) or cell death (PI positive staining).

\section{Effects of essential oil on fibroblast proliferation}

The exposure of fibroblasts to EO at a concentration of $10 \%$ caused a higher increase in the number of cells in the dishes both after 24- and 48-hour culture as compared with the control group, but the difference was not significant: $111.8 \pm 14.9 \%$ vs $112.4 \pm 1.3 \%(p=0.3)$ and $139.5 \pm 29.5 \%$ vs. $137.4 \pm 5.8 \%(p=0.8)$, respectively. Essential oil at concentrations of $50 \%$ and $100 \%$ added to the culture medium caused a significant decrease in the number of cells in the culture dishes both after 24 hours $(78.3 \pm 14.4 \%$ and $52.8 \pm 9.6 \%$, respectively) and 48 hours $(50.7 \pm 18.0 \%$ and $33.7 \pm 20.8 \%$ ) of culture. The differences were significant both in comparison with the control group $(p<0.001)$ and with cells cultured with EO 10, $p<0.001$ (Fig. 2). After 48-hour-culture with EO 50 or EO 100 the percentage of dead or apoptotic cells was $12.1 \pm 6.2 \%$ and $29.2 \pm 11.3 \%$, respectively $(p<0.05)$.

\section{Effects of salvia on fibroblast proliferation}

Salvia caused a gradual decrease in the number of cells on the plates. In comparison with the control group, both after 24 and 48 hours of culture, the difference was significant: $77.1 \pm 1.81 \%$ vs $112.4 \pm 1.3 \%(p<0.001)$ and $45.6 \pm 17.4 \%$ vs $137 \pm 5.8 \%$ ( $p=0.001)$, respectively. After 48 hours of culture with salvia, the percentage of dead or apoptotic cells was $15.4 \pm 8.2 \%$.

\section{Effects of calendula on fibroblast proliferation}

After 24 and 48 hours of culture of fibroblasts in the medium with calendula, a gradual increase in the number of cells on the plates could be observed. The increase was slightly higher than in the control group: $146.6 \pm 35.7 \% \mathrm{vs}$ $112.4 \pm 1.3 \%(p=0.14)$ and $183.2 \pm 60.5 \%$ vs $137.4 \pm 5.8 \%$ $(p=0.13)$, respectively, the difference was not significant. No apoptotic structural changes were observed after 48 hours of culture.

\section{Effects of chamomile on fibroblast proliferation}

After 24 and 48 hours of the culture of fibroblasts in the medium with chamomile, an increase in the number of cells on the plates could be observed. However, it was slightly lower than in the control group: $101.8 \pm 13.6 \% \mathrm{vs}$ $112.4 \pm 1.3 \%(p=0.14)$ and $118.6 \pm 39.8 \%$ vs $137.4 \pm 5.8$ $\%(p=0.34)$, respectively. No apoptotic structural changes were observed after 48 hours of cell culture.

Chlorhexidine and EO at the concentrations of $50 \%$ and $100 \%$ and salvia caused a significant decrease in the relative number of cells on Petri plates. The comparison of these substances proved that both after 24 and 48 hours of culture, the highest decrease could be observed in media with salvia and EO $100(p<0.001)$. The effects caused by CHX and EO 50 after 24 hours of incubation did not differ significantly $(p>0.05)$, but after 48 hours the decrease in the number of cells was significantly higher with EO 50 than with CHX, $p<0.05$ (Fig. 2A and B).

\section{Discussion}

For many years antiseptic rinses have been successfully used in inflammatory conditions of the periodontal tissues and oral cavity mucosa. Their efficacy has been confirmed in clinical and laboratory investigations. EO contains a fixed combination of 4 essential oils as active ingredients (thymol, $0.064 \%$; eucalyptol, $0.092 \%$; methyl salicylate, $0.060 \%$; menthol, 0.042) and kills microorganisms by disrupting their cell walls and inhibiting enzymatic activity [11]. Essential oil reduces the possibility of infection with herpes viruses HHV-1 or HHV-2 and the influenza virus by nearly $100 \%$ [12]. Essential oils prevent bacterial aggregation, slow bacterial multiplication, and extract endotoxins [13]. In order to evaluate the influence of EO on fibroblasts three concentrations of the rinse were used: $10 \%, 50 \%$ and $100 \%$. The obtained results indicate that growth inhibition is proportional to the applied concentrations. Essential oil 10\% caused a slight cell growth, 

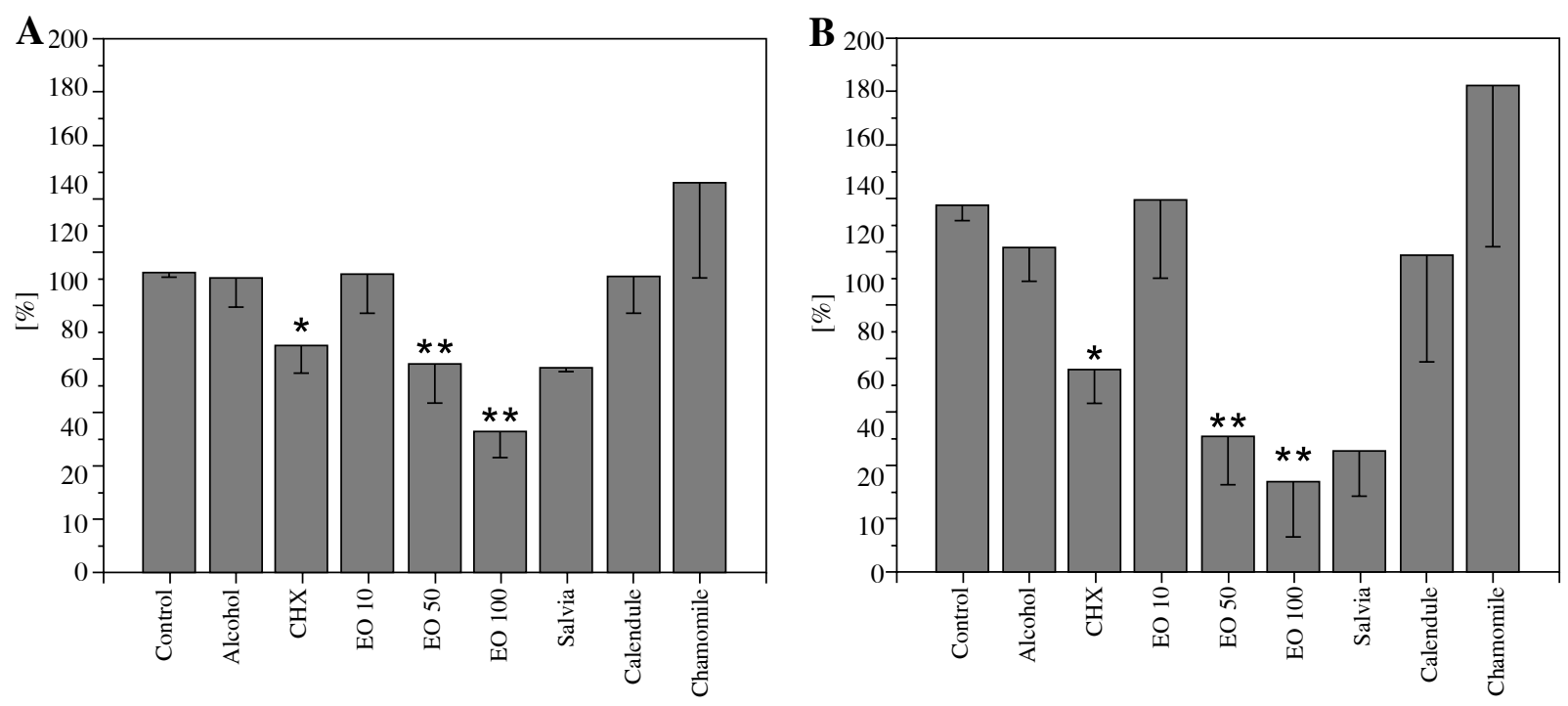

* statistically significant differences: CHX vs. control

** EO 50, EO 100 vs. control and EO 10.

Fig. 2. The effect of alcohol, chlorhexidine $0.1 \%$ (CHX), essential oils (EO) in dilution of 10\% (EO 10), 50\% (EO 50), $100 \%$ (EO 100) and salvia, chamomile or calendula brews on the relative changes of the number of cells in percent after 24 (A) and 48 (B) hours of culture

whereas the solutions of $50 \%$ and $100 \%$ caused a considerable decrease in the cell number. Similar results were shown by Flemingson et al. [14]. It is necessary to stress that the cell morphology was not changed. Simultaneously, the clinical and morphohistological studies on the tissue healing process by Kozlovsky et al. [15] stress the absence of inhibition of epithelial cell proliferation by EO 100\%, which contradicts results we obtained.

Chlorhexidine bigluconate efficiently eliminates Gram-positive and Gram-negative bacteria, their vegetative forms, yeasts, fungi, protozoa as well as viruses. It modulates the metabolism of Candida fungi and causes reduction of their pathogenicity [16]. Due to the interaction between chlorhexidine bigluconate molecules and the bacterial wall and the mucoproteins of the mucin layer it maintains prolonged action in the environment of the mouth. $0.1 \% \mathrm{CHX}$ is considered to be one of the most effective substances preventing the development of pellicle [11]. The ability of chlorhexidine to non-selectively kill oral microbiota also makes it an excellent agent to indiscriminately affect mammalian cells. In fact, the effect of chlorhexidine on a variety of mammalian cells has shown this drug to be a toxic agent at doses similar to below those introduced into the oral cavity [17]. The results we obtained point to the negative influence of $\mathrm{CHX}$ both on cell proliferation and morphology. Cell apoptosis and contraction were observed. Shakespeare et al. [18] obtained similar results in their studies. The studies comparing the antibacterial effect of EO and CHX indicate that both mouth rinses showed considerable antimicrobial effects on the monospecies biofilm in vitro. EO showed a stronger bactericidal effect but had a weaker bacterial inhibitory effect than CHX [11]. Due to the fact that both rinses contain ethyl alcohol, the influence of $10 \%$ alcohol on fibroblasts was also examined. Alcohol varies in the concentration used in products, from typical of around $7 \%$ to greater of $25 \%$ [19]. On administration, cell contraction could be observed. After 24 hours their shape was close to normal, but they had a reduced number of projections and the total cell count was slightly higher. The obtained results indicate that $10 \%$ alcohol does not inhibit fibroblast proliferation. According to Sliepen et al., the ethanol solution at a concentration below $30 \%$ did not show antimicrobial effects on $S$. mutans biofilms [20]. The presence of alcohol in a mouth rinse containing $0.10 \% \mathrm{CHX}$ has no deleterious effects on healing capacity. On the contrary, it helps stimulate wound healing [21]. The combination of CHX plus alcohol is superior for healing, $\mathrm{CHX}$ alone does not show any significant difference compared with the control [21].

On the other hand, natural products with anti-inflammatory properties, such as green tea, aloe, manuka or tea tree oil, are more and more frequently used in ready-made mouth rinses [22, 23]. Clinical tests confirm their high efficacy. Although less potent than Peridex, the Natural Dentist Healthy Gums Oral Rinse, containing aloe vera, was a more effective antimicrobial than EO in inhibiting the growth of oral bacteria in vitro [5]. Streptococcus and Capnocytophaga species were reduced most in the herbal rinse 
groups, however Veillonella parvula was reduced most in the essential oil and chlorhexidine groups [4]. Herbal extracts mostly have the anti-inflammatory effect.

Chamomile (Matricaria chamomilla L.) is one of the important herbs in medicine with pharmacological properties. They show the anti-inflammatory, antiseptic, carminative, healing, sedative and spasmolytic activity [24]. The active compounds in the chamomile are $\alpha$-bisabolol and spiroether, which inhibit the secretion of histamine, serotonin and bradykinin. In vitro and in vivo studies report the fact that chamomile causes wounds to heal sooner than corticosteroid treatment [25]. The herb stimulates faster formation of the epithelium [26]. Our studies proved that it does not influence the fibroblast morphology.

Apart from its anti-inflammatory and antibiotic effect, the calendula reduces the permeability of capillary walls. It accelerates healing of wounds [27]. However, it is a weaker inhibitor of adherence of microorganisms than $\mathrm{CHX}$ [28]. When the calendula brew was applied, favourable morphological changes in the cell structure could be observed. They were manifested by numerous and elongated projections and an increased number of cells. This fact is confirmed by the studies [29] which say that extracts of Calendula officinalis stimulated the proliferation and migration of fibroblasts at low concentrations.

For centuries salvia brew has been used as a disinfectant and astringent. The experiments by Al-Bakri et al. [30] point to the antibacterial effect of some of salvia varieties. Ursolic acid is a component of salvia. The anti-inflammatory effect of ursolic acid (ID50 $=0.14 \mu \mathrm{M} / \mathrm{ml}$ ) was twice as strong as that of indometacin (ID50 $=0.25 \mu \mathrm{M} / \mathrm{ml}$ ) used as a non-steroidal anti-inflammatory reference compound (NSAID) [31]. The applied salvia brew had negative influence on the number of cells and their morphology. A considerable part of the fibroblasts was contracted and the cells had few, narrow and elongated projections. The negative influence of salvia on fibroblast metabolism was also observed by He et al. [32] who noticed the inhibition of collagen synthesis in the cells.

\section{Conclusions}

The study demonstrated that certain components of antiseptic rinses used in inflammation of gums and oral mucosa, apart from their undoubted antibacterial action, can negatively affect biology of human fibroblasts in vitro. Oral cavity fibroblasts represent a group of heterogenic cells and their reaction to antiseptic rinses requires further research. Considering our results it seems possible that permanent or high concentration application can lead to atrophic changes of periodontal tissue. Chamomile and calendula extracts with strong antibacterial properties were the only mouth rinses that did not show anti-proliferative effects on fibroblast cells and in calendula case accelerates healing of wounds.
The authors decvlare no conflict of interests.

This study was supported by the Poznan University of Medical Sciences research grant (50201-04410519006466).

\section{References}

1. Cochran DL (2008): Inflammation and bone loss in periodontal disease. J Periodontol 79: 1569-1576.

2. Forabosco A, Spinato S, Grandi T, et al. (2006): A comparative study between different techniques in non-surgical periodontal treatment. Minerva Stomatol 55: 289-296.

3. Lang NP, Tan WC, Krahenmann MA, et al. (2008): A systematic review of the effects of full-mouth debridment with and without antiseptics in patients with chronic periodontitis. JClin Periodontol 35: 8-21.

4. Haffajee AD, Roberts C, Murray L, et al. (2009): Effect of herbal, essential oil, and chlorhexidine mouthrinses on the composition of the subgingival microbiota and clinical periodontal parameters. J Clin Dent 20: 211-217.

5. Haffajee AD, Yaskell T, Socransky SS (2008): Antimicrobial effectiveness of an herbal mouthrinse compared with an essential oil and a chlorhexidine mouthrinse. J Am Dent Assoc 139: 606-611.

6. Wu CD, Savitt ED (2002): Evaluation of the safety and efficacy of over-the-counter oral hygiene products for the reduction and control of plaque and gingivitis. Periodontol 2000 28: 91-105.

7. Claffey N (2003): Essential oil mouthwashes: a key component in oral health management. J Clin Periodontol 30 Suppl 5: 22-24.

8. Seymour R (2003): Additional properties and uses of essential oils. J Clin Periodontol 30 Suppl 5: 19-21.

9. Mariotti A, Cochran DL (1990): Characterization of fibroblasts derived from human periodontal ligament and gingiva. J Periodontol 61: 103-111.

10. Mitrano TI, Grob MS, Carrion F, et al. (2010): Culture and characterization of mesenchymal stem cells from human gingival tissue. J Periodontol 81: 917-925.

11. Chen Y, Wong RW, Seneviratne CJ, et al. (2011): Comparison of the antimicrobial activity of Listerine and Corsodyl on orthodontic brackets in vitro. Am J Orthod Dentofacial Orthop 140: 537-542.

12. Dennison DK, Meredith GM, Shillitoe EJ, et al. (1995): The antiviral spectrum of Listerine antiseptic. Oral Surg Oral Med Oral Pathol Oral Radiol Endod 79: 442-448.

13. Ouhayoun JP (2003): Penetrating the plaque biofilm: impact of essential oil mouthwash. J Clin Periodontol 30 Suppl 5: 10-12.

14. Flemingson, Emmadi P, Ambalavanan N, et al. (2008): Effect of three commercial mouth rinses on cultured human gingival fibroblast: an in vitro study. Indian J Dent Res 19: 29-35.

15. Kozlovsky A, Artzi Z, Hirshberg A, et al. (2007): Effect of local antimicrobial agents on excisional palatal wound healing: a clinical and histomorphometric study in rats. J Clin Periodontol 34: 164-171.

16. Ellepola AN, Joseph BJ, Khan ZU (2012): Effects of subtherapeutic concentrations of chlorhexidine gluconate on germ tube formation of oral Candida. Med Princ Pract 21: 120-124. 
17. Louis SM, Pearson RM (1985): A comparison of the effect of nonoxylon-9 and chlorhexydine on sperm mobility. Contraception 32: 199-205.

18. Shakespeare V, Shakespeare PG, Evans BT (1988): Effects of proprietary oral rinses containing chlorhexidine, hexetidine and benzydamine on the proliferation of human buccal epithelial cells in culture. Arch Oral Biol 33: 881-885.

19. Addy M (2008): Oral hygiene products: potential for harm to oral and systemic health? Periodontol 2000 48: 54-65.

20. Sliepen I, Van Essche M, Quirynen M, et al. (2010): Effect of mouthrinses on Aggregatibacter actinomycetemcomitans biofilms in a hydrodynamic model. Clin Oral Investig 14: 241-250.

21. Boisnic S, Ben Slama L, Branchet-Gumila MC, et al. (2006): Wound healing effect of Eludril in a model of human gingival mucosa. Rev Stomatol Chir Maxillofac 107: 431-435.

22. Arweiler NB, Donos N, Netuschil L, et al. (2000): Clinical and antibacterial effect of tea tree oil - a pilot study. Clin Oral Investig 4: 70-73.

23. Lauten JD, Boyd L, Hanson MB, et al. (2005): A clinical study: Melaleuca, Manuka, Calendula and green tea mouth rinse. Phytother Res 19: 951-957.

24. Salamon I (1992): Chamomile a medicinal plant. J Herb, Spice Med Plant: 1-4.

25. Martins MD, Marques MM (2009): Comparative analysis between Chamomilla recutita and corticosteroids on wound healing. An in vitro and in vivo study. Phytother Res 23, 2748 23: 274-278.

26. Duarte CM, Quirino MR, Patrocinio MC, et al. (2011): Effects of Chamomilla recutita (L.) on oral wound healing in rats. Med Oral Patol Oral Cir Bucal 16: e716-721.

27. Magee K, Loiacono C (2004): A review of common herbs and potential interactions. Int J Dent Hyg 2: 111-121.

28. Faria RL, Cardoso LM, Akisue G, et al. (2011): Antimicrobial activity of Calendula officinalis, Camellia sinensis and chlorhexidine against the adherence of microorganisms to sutures after extraction of unerupted third molars. J Appl Oral Sci 19: 476-482.

29. Fronza M, Heinzmann B, Hamburger M, et al. (2009): Determination of the wound healing effect of Calendula extracts using the scratch assay with $3 \mathrm{~T} 3$ fibroblasts. J Ethnopharmacol 126: 463-467.

30. Al-Bakri AG, Othman G, Afifi FU (2010): Determination of the antibiofilm, antiadhesive, and anti-MRSA activities of seven Salvia species. Pharmacogn Mag 6: 264-270.

31. Baricevic D, Sosa S, Della-Loggia R, et al. (2001): Topical anti-inflammatory activity of Salvia officinalis L. leaves: the relevance of ursolic acid. J Ethnopharm 75: 125-132.

32. He S, Yang Y, Liu X, et al. (2012): Compound Astragalus and Salvia miltiorrhiza extract inhibits cell proliferation, invasion and collagen synthesis in keloid fibroblasts by mediating transforming growth factor-beta / Smad pathway. $\mathrm{Br}$ J Dermatol 166: 564-574. 